\title{
Perception of Ultrasonic Vocalizations by Socially Housed and Isolated Mice
}

\author{
Laurel A. Screven and Micheal L. Dent
}

https://doi.org/10.1523/ENEURO.0049-19.2019

Department of Psychology, University at Buffalo, the State University of New York, Buffalo, NY 14260

\begin{abstract}
It is currently unclear whether mice use their ultrasonic vocalizations (USVs) for communication purposes. It is also unknown whether mice require previous experience with USVs to understand conspecifics. There is some evidence that experience changes the perception of juvenile USVs; however, it is unclear whether similar plasticity also occurs for adult USVs. To examine whether social exposure or deprivation throughout development leads to changes in USV perception, eleven female CBA/CaJ mice were trained to discriminate between 18 USVs of three different categories using operant conditioning procedures. Mice were group housed with four females or housed individually from weaning for the duration of the experiment. Socially housed and isolated mice differed in initial training times on pure tones, suggesting isolated mice had a more difficult time learning the task. Both groups completed USV discrimination conditions quicker at the end of the testing phases relative to the beginning. The overall discrimination of USVs did not differ between the two housing conditions, but a multidimensional scaling analysis revealed that socially experienced and isolated mice perceive some USVs differently, illustrated by differences in locations of USVs on the scaling maps from the two groups. Finally, a negative correlation was found between spectrotemporal similarity and percent discrimination, and analyses support the idea that mice may show categorical perception of at least two of the three USV categories. Thus, experience with USVs changes USV perception.
\end{abstract}

Key words: hearing; psychoacoustics; USVs

\section{Significance Statement}

The present experiment is the first to behaviorally measure changes in perception of ultrasonic vocalizations (USVs) by mice after different levels of social experience. Electrophysiological experiments showed increases in cortical spiking in maternal females in response to pup calls (Liu and Schreiner, 2007; Cohen et al., 2011), but it is unknown whether changes in neuronal activity are correlated with changes in perception. Psychophysical measurements of USV perception from awake, behaving mice with different social experiences are sorely needed. Further, USVs have historically been divided into researcher-defined categories, and the present experiment is the first to examine whether mice exhibit categorical perception of adult USVs. It is critical to ascertain the factors that influence auditory perception to further our understanding of mouse communication.

\section{Introduction}

For vocalizations to be useful for communication, it is critical to perceive and understand the various vocal signals emitted by conspecifics. Mice emit ultrasonic vocalizations

Received February 12, 2019; accepted September 10, 2019; First published September 30, 2019

The authors declare no competing financial interests.
(USVs), which differ in spectrotemporal parameters (e.g., frequency, duration, and intensity). USVs are produced in same- and opposite-sex interactions by both males and females (Sewell, 1972; Scattoni et al., 2011; Hammer- 
schmidt et al., 2012; Neunuebel et al., 2015) and are assumed to facilitate social interactions (Portfors, 2007; Okanoya and Screven, 2018).

USVs have been divided into categories based on spectrotemporal parameters (Sewell, 1972; Portfors, 2007; Scattoni et al., 2011; Hammerschmidt et al., 2012), but it is currently unknown whether these categories are meaningful to the mice. Due to the spectrotemporal variability of USVs, individual USVs could possibly have a situation-specific function in mice, where certain USVs or sequences communicate information in particular situations. To determine whether USVs have context specificity, we must first ask whether mice can discriminate between the various USVs they produce. When mice are able to discriminate between USVs, the USVs have the potential to communicate contextspecific information.

Auditory processing is often examined using electrophysiological methods, such as extracellular recordings (Portfors et al., 2009). Electrophysiology does not require training, but it often yields measures that are less informative than results from awake, behaving mice (Klink et al., 2006; Heffner et al., 2008). It is impossible to determine whether differences in neural responsivity correspond to actual differences in perception by the animal. Through operant conditioning, mice are trained to be "reliable observers" (Heffner and Heffner, 2001, p 19) and researchers can make more accurate and nuanced measures of their perceptual abilities (Dent et al., 2018). For example, Neilans et al. (2014) found that mice could discriminate between different categories of USVs, with increased discrimination for spectrotemporally dissimilar vocalizations. That experiment was the first to show that mice could discriminate between USVs of different researcher-defined categories. The findings of that experiment are limited, however, because only one USV from each researcher-defined category was used. The relationship between discrimination and spectrotemporal similarity could be more complex than was reported by Neilans et al. (2014).

Perception of USVs may be affected by social experience. Neilans et al. (2014) used chronically socially isolated mice, and isolation could have led to deficits in discrimination. Mice may need to be exposed to USVs through social experience throughout development for those USVs to have a communicative function (see Chabout et al., 2015, 2016). The role of social experience with USVs on the spiking activity of neurons in the mouse auditory cortex was examined by Liu and Schreiner

This work was supported by the National Institutes of Health Grant DC012302 (to M.L.D.).

Acknowledgements: We thank Nina Baldy, Anastasiya Kobrina, Kali Burke, Ethan Gorman, and numerous undergraduates for their assistance.

Correspondence should be addressed to Micheal L. Dent at mdent@buffalo.edu.

https://doi.org/10.1523/ENEURO.0049-19.2019

Copyright (C) 2019 Screven and Dent

This is an open-access article distributed under the terms of the Creative Commons Attribution 4.0 International license, which permits unrestricted use, distribution and reproduction in any medium provided that the original work is properly attributed.
(2007). There were significant increases in cortical spiking activity in maternal compared to virgin female mice to pup USV playbacks. Cohen et al. (2011) extended these findings to include pup-experienced virgin females, and showed similar spiking activity as in maternal females. This increase in spiking activity arose presumably because of the behavioral relevance of these USVs to the pup-experienced virgin and maternal females compared to pup-naive virgin females. It is unclear from these findings whether there were perceptual differences to pup calls as a result of differences in spiking activity since no behavioral measurements were taken.

Social interactions could be critical for mice to discriminate vocalizations emitted by conspecifics. Social experiences of female mice are known to influence the preference for USVs and olfactory signals (Screven and Dent, 2018), but not the production of USVs (Screven and Dent, 2019). Preventing mice from learning the connections between vocalizations and context could be detrimental to their ability to perceive USVs although it does not change the production of the USVs. To investigate the effect of social deprivation on USV perception, we compared USV discrimination in socially housed and chronically isolated mice. We hypothesized that exposure to conspecifics through social housing would improve USV discrimination compared to discrimination by isolated mice. Additionally, we hypothesized that chronic social isolation would lead to irreversible perceptual deficits. There was a difference in training time between mice in the two social housing conditions in early training on pure tones, but not on later overall USV discrimination abilities, although a multidimensional analysis revealed differences in perceptual mapping of the USVs between the two groups. Finally, we were able to expand on the findings of Neilans et al. (2014) by examining the role of spectrotemporal similarity of USVs on discrimination performance, and found that the mice perceived at least some of the USVs in a categorical manner.

\section{Materials and Methods}

\section{Animals}

Eleven female CBA/CaJ mice were used for this experiment. These mice were divided into two groups: individually housed and socially housed. All five individually housed mice were experimentally naive. Two socially housed female mice (Js and $\mathrm{Fr}$ ) were experimentally naive; the remaining four (Jo, Ja, Re, and Fa) were previously tested on a similar experiment investigating USV perception. That experiment was an exploratory test to examine whether mice could more easily discriminate between USVs from a familiar versus an unfamiliar mouse. The two socially housed mice that were experimentally naive provided a control for the other mice's previous experience with the test stimuli and procedure. The individually housed mice were separated from their litter at weaning and lived alone in their home cages $(30 \times 19 \times 13 \mathrm{~cm})$ with stainless steel covers, bedding, and nesting material for the duration of the experiment. These mice did not have any social contact with any other mice, although they were not acoustically isolated from the rest of the 


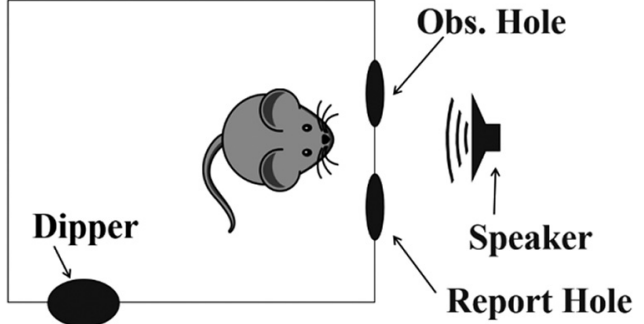

Figure 1. Schematic of the operant conditioning chamber. Mice were trained to nose poke into the observation (obs.) and report holes to receive reinforcement from the dipper.

colony. The six socially housed mice were housed in two large home cages $(47 \times 25.5 \times 21.5 \mathrm{~cm})$ with stainless steel covers, bedding, and nesting material. Socially housed mice lived in groups of four. Re, Ja, Jo, and Fa lived together in cage 1 and $\mathrm{Fr}$ and Js lived in cage 2, along with two mice used in other experiments.

\section{Apparatus}

The mice were tested in a wire cage $(23 \times 39 \times 15.5$ $\mathrm{cm})$ placed in a sound attenuated chamber $(53.3 \times 54.4 \times$ $57 \mathrm{~cm}$ ) lined with 4-cm thick Sonex sound attenuating foam (Illbruck, Inc.). The chamber contained an overhead web camera (Logitech B910 HD) and a small 25-W white light to monitor mice during test sessions. Signals were played from an electrostatic speaker (Tucker-Davis Technologies, Model ES1). The cage also contained two nose-poke holes surrounded by infrared sensors (Med Associates Model ENV-254), and a response dipper (Med Associates Model ENV-302M-UP; Fig. 1).

\section{Test stimuli}

Vocalizations were recorded from two female CBA/CaJ mice (Mouse $\mathrm{K}$ and Mouse I) who previously (approximately six months earlier) lived with the four experimentally experienced mice (two groups of four, one vocalizer per cage). Three mice lived with one vocalizer ( $\mathrm{Re}, \mathrm{Ja}$, and Jo lived with Mouse K), and the remaining mouse lived with the other (Fa lived with Mouse I). The mice used in this experiment did not live with the vocalizers at any point during testing. Vocalizations were recorded using a condenser microphone [UltraSoundGate CM16/CMPA, flat frequency response $( \pm 6 \mathrm{~dB})$ between 25 and $140 \mathrm{kHz}$, which was attached to the lid of the recording chamber, 8 $\mathrm{cm}$ above the cage. Acoustic signals traveled to an HP Pavilion 500 PC computer through an Avisoft recorder (BioAcoustics UltraSoundGate $116 \mathrm{H}, 300-\mathrm{kHz}$ sampling rate with a 16-bit format). Vocalizations were analyzed with Adobe Audition CS6 on an HP Pavilion 500 PC.

The stimuli were recorded by placing one female in a home cage $(18.5 \times 12.5 \times 29.5 \mathrm{~cm})$ that contained dirty bedding from a cage mate. The cage was placed inside a recording chamber $(46 \times 41 \times 74 \mathrm{~cm})$. This chamber was lined with Sonex anechoic foam $(4 \mathrm{~cm})$. Vocalizations were elicited from the mice by putting a cage mate in a separate home cage $14 \mathrm{~cm}$ away, following a 6-h separation. Due to the high directionality of the USVs, we were able to ensure all USVs in the recording were from the desired female, as USVs from the other female were much quieter on the recording.

The recordings were analyzed and we chose USV s from three categories to use as stimuli in this experiment. These categories were chevron, complex, and upsweep (Fig. 2). A total of 18 USVs were used as stimuli: five chevron, six complex, and seven upsweep USVs. Chevron USVs were characterized by an inverted U-shape in frequency modulation. They had a mean $20-\mathrm{kHz}$ rise and $13-\mathrm{kHz}$ fall in frequency and a mean duration of $83 \mathrm{~ms}$. Complex USVs had a minimum of two changes in the direction of frequency modulation and frequency modulations of at least $5 \mathrm{kHz}$. These USVs had a mean duration of $60 \mathrm{~ms}$. Upsweep USVs were characterized by an increase in frequency across the duration of the USV, with a mean total increase of $20 \mathrm{kHz}$. Upsweep USVs may show a slight decrease in frequency at the end of the USV, but the end frequency of the stimulus must be $>7 \mathrm{kHz}$ from the starting frequency of the stimulus. Therefore, these upsweep USVs are distinct from chevron USVs. Additionally, upsweep USVs may show a slight decrease in frequency at the beginning of the USV. The upsweep USVs had a mean duration of $51 \mathrm{~ms}$. All USVs were presented at the same intensity [50-dB SPL, LAFmax (maximum intensity of a sound rising using the A-scale and fast time constant)] during the entirety of the experiment.

The stimuli were named, for example, with the nomenclature of: "I Chevron B", where "I" refers to the vocalizer (I or K), "Chevron" refers to the category of USV (chevron, complex, or upsweep), and "B" refers to the rendition ( $A-D$, a random assignment of different vocalizations fitting the category produced by the specific vocalizer).

\section{Experimental design and statistical analyses}

Mice were trained using a go/no-go operant conditioning procedure on a discrimination task. The mice were tested once per day for $1 \mathrm{~h}, 7 \mathrm{~d}$ per week. Each mouse was tested on all 18 USVs unless they became too ill to complete the experiment. Each USV served as a background and target stimulus. Every background-target stimulus combination was tested for each mouse.

Before the mice began testing, they were trained using strict criteria for both hit rate and false alarm rate on a pure tone detection task. The training stimulus was a $500-\mathrm{ms} 16-\mathrm{kHz}$ pure tone with a $40-\mathrm{ms}$ rise/fall time. Pure tones were presented two times, separated by $500 \mathrm{~ms}$ of silence, and mice were required to correctly respond to tones before being tested using USVs. After mice reliably detected the $16-\mathrm{kHz}$ pure tone, they were transitioned to USV stimuli. To ensure that the mice understood the discrimination procedure, they were first trained to detect high-intensity target USVs while the background USV was attenuated to $0-\mathrm{dB}$ SPL. When the subjects were able to detect over $80 \%$ of the targets correctly with less than a $20 \%$ false alarm rate (criterion performance), the background attenuation decreased by $5-10 \mathrm{~dB}$ until the same criterion performance was reached again, then the background was attenuated less, and so on. This continued until the background USV was presented at the same 


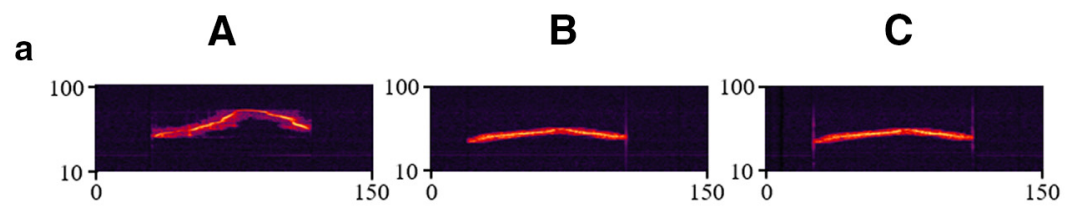

b

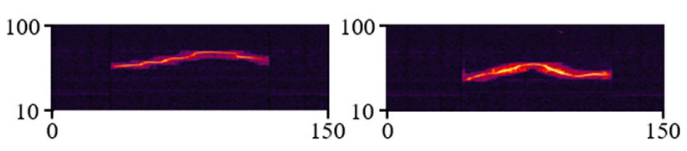

C

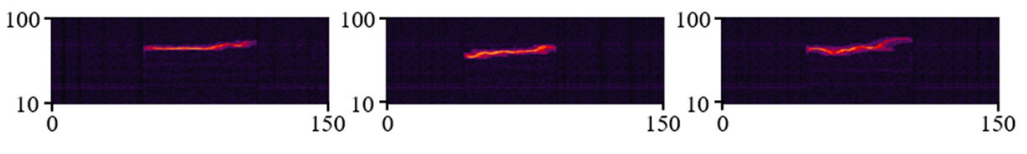

d

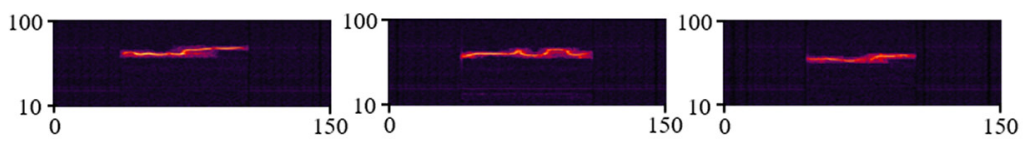

e

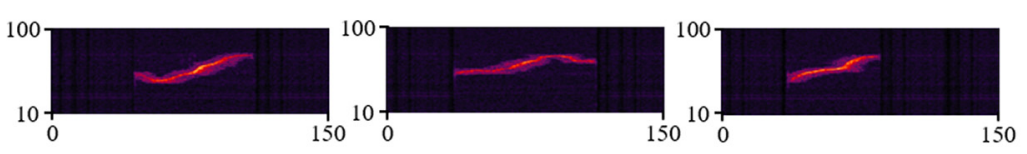

f

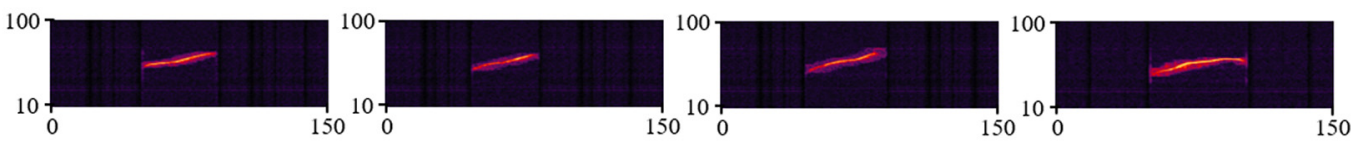

\section{Time (ms)}

Figure 2. Spectrograms of all USVs used in this experiment. USVs in rows a, $\boldsymbol{c}$, e were produced by Mouse K, while USVs in rows $\boldsymbol{b}, \boldsymbol{d}, \boldsymbol{f}$ were produced by Mouse I. Chevron vocalizations are depicted in rows $\boldsymbol{a}, \boldsymbol{b}$. Complex vocalizations are depicted in rows $\boldsymbol{c}$, d. Upsweep vocalizations are depicted in rows e, $\boldsymbol{f}$. $\boldsymbol{A}-\boldsymbol{D}$ are different renditions of each USV.

intensity as the target USVs, at which time the mouse was considered in the testing phase.

Mice began a trial by nose poking through the left observation hole, which initiated a variable waiting interval that ranged from 1 to $4 \mathrm{~s}$. During this time, one of the 18 USVs that served as the background stimulus was presented repeatedly, with a 200-ms silence interval between each presentation. Only one background USV was presented per session. After the waiting interval, one of seven possible target USVs was presented, alternating with the background two times. When the mouse was able to discriminate between the background and the target USVs, it was required to nose poke through the right report nose-poke hole within $2 \mathrm{~s}$ of the onset of the test stimulus. In this trial type, a "hit" was recorded when the mouse responded within the response window and the mouse received $0.01 \mathrm{ml}$ of Ensure as reinforcement. A "miss" was recorded when the mouse failed to nose poke through the report nose-poke hole during the response interval. In this case, the mouse was able to move on to the next trial immediately, as no punishment (timeout) was administered when the mouse missed a target stimulus. A schematic of the trial structure is shown in Figure 3.
Experimental sessions consisted of multiple random blocks of ten trials each. Within each block of ten, seven were "go" trials and three were "no-go" trials. The sequence of go and no-go trials within each block was random except for the constraint that no more than two no-go trials were presented in a row. In the no-go trials, the repeating background continued to be presented during the response phase. These trials were required to measure false alarm rate to determine whether the mouse was guessing. When the subject nose poked in the report poke-hole during the response period, a "false alarm" was recorded and the mouse was punished with a 5-s timeout interval, during which another trial could not be initiated. When the subject continued to nose poke into the observation hole, a "correct rejection" was recorded and the mouse continued on to the next trial immediately (no timeout). In either case, no reinforcement was given. False alarm rate is a measure of how often mice randomly responded into the report hole when no target USVs were presented. Sessions were excluded from analysis when the mice's false alarm rate exceeded $20 \%$.

In go trials, seven of the seventeen possible target USV types were presented randomly throughout the session. When a mouse completed 20 trials for each of the first 


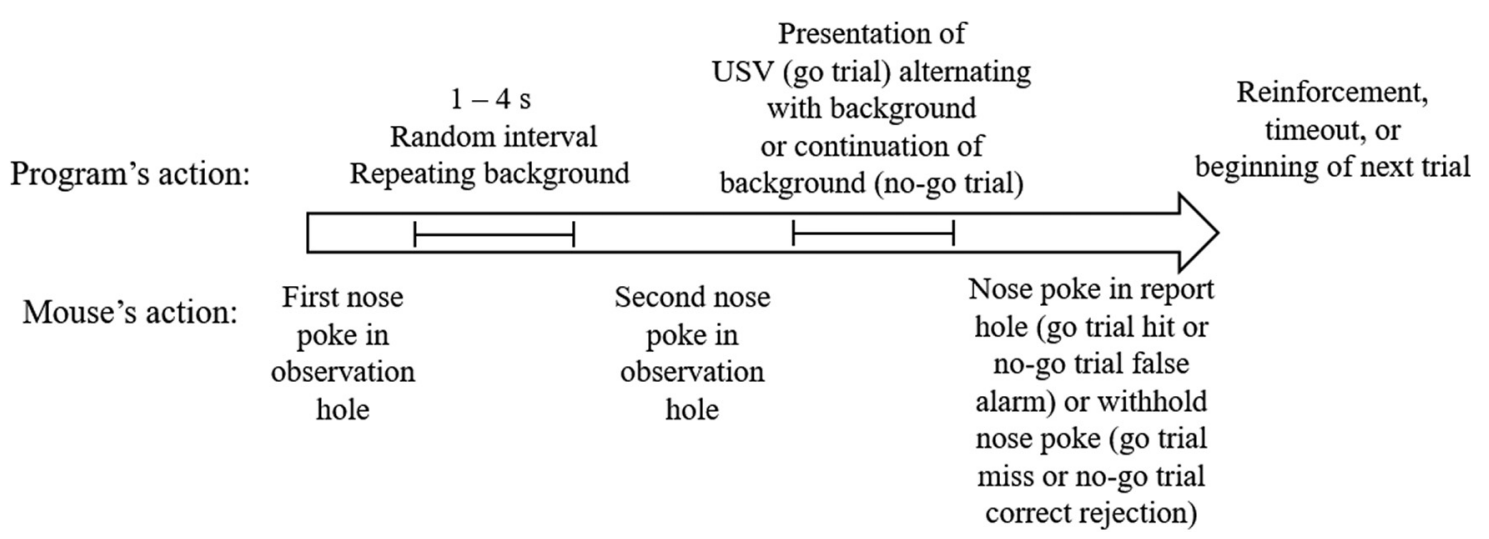

Figure 3. Schematic of the trial structure of the USV discrimination task.

seven USV types (200 total trials including the 60 no-go trials) with a false alarm rate $<20 \%$, the mouse remained on the same background USV and the next seven USV types were used as target stimuli. Mice were not required to reach a minimum percent correct of $80 \%$ during the testing phase, as mice were not able to discriminate some targets from the background during testing. After the mouse completed 200 trials for the second group of target stimuli, they were tested on the last three USVs with the same USV serving as the background as the previous two conditions and completed 20 trials for each of the remaining 3 USVs (100 total trials, including 30 no-go trials and 10 already tested USV trials). A mouse completed a condition once all 17 target USVs had been discriminated from the background USV at least 20 times each. All mice were tested on all background-target USV combinations in a random order, and a different random order was used for each subject. Mice completed between 50 and 300 trials per session.

To determine whether there were cognitive deficits in isolated mice, we conducted a Mood's median test to compare the number of training days required by the isolated and socially housed mice before they could begin testing. We also conducted a Mood's median test to evaluate the relationship between condition number and number of days required to complete each condition between socially housed and isolated mice. It is important to note that condition number refers to the order the mice ran on each condition, regardless of which USV served as the background. To correct for multiple comparisons, a Bonferroni correction was applied such that significant results are denoted by $p<0.003$. Additionally, to determine whether there were differences in discrimination of target USVs from background USVs between social and isolated mice, we conducted a Mann-Whitney $U$ test on the percent correct for each target USV separately, using housing condition (social vs isolated) as the factor of comparison. A Bonferroni post hoc correction was applied to correct for multiple comparisons, such that significant differences are denoted by $p<0.003$. Next, we conducted a multidimensional scaling analysis (PROXSCAL, identity), which used percent discrimination of every USV versus every other USV to determine the perceptual maps of the USVs for social and isolated female mice. To examine how each mouse contributed to the multidimensional scaling analyses, we created an individual weight graph (PROXSCAL, weighted Euclidian). To determine the relationship between discrimination performance and spectrotemporal similarity of USVs, we conducted Spearman's rank-order correlations for social and isolated mice. Lastly, to examine whether mice differentially discriminated USVs within versus between categories, we conducted three Mann-Whitney $U$ tests on discrimination performance (percent correct) for all three background USV categories (within category vs between categories) for both socially housed and isolated mice. To correct for multiple comparisons, a Bonferroni correction was applied such that significant results are denoted by $p<0.008$.

\section{Results}

\section{Number of training and testing days per condition}

A Mood's median test revealed significant differences between socially housed mice and socially isolated mice with respect to number of days required to train on pure tone stimuli $\left(\chi^{2}=4.412, p=0.036\right)$. Socially housed mice required a median of $50 \mathrm{~d}$ to complete training, significantly fewer days than isolated mice, which required $97 \mathrm{~d}$ (Fig. 4). This effect is not the result of the four social mice ( $\mathrm{Jo}, \mathrm{Ja}, \mathrm{Re}$, and $\mathrm{Fa}$ ) having experience with another USV perception task before the beginning of the present experiment, as the training took place before the onset of all USV discrimination tasks for all mice in both housing groups. Next, the number of days required to complete each USV discrimination condition was examined in both housing groups (Fig. 5). The results of the Mood's median test revealed isolated mice required significantly more days to complete the first condition than socially housed mice $\left(\chi^{2}=11.000, p=0.0009\right.$; regardless of which USV served as the background). No other condition showed significant differences between mice in the two housing conditions. Thus, initially, socially housed mice performed better on the discrimination task than isolated mice, but after the first condition both groups required a similar number of days to complete each condition. 


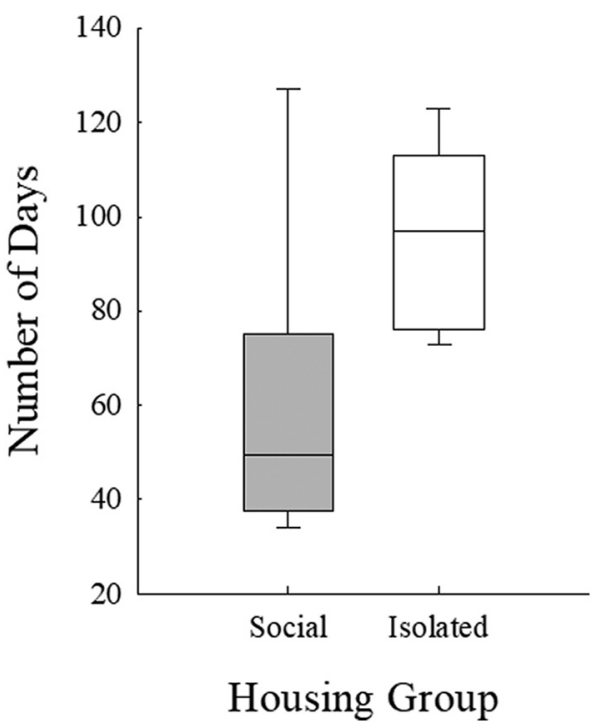

Figure 4. Number of days required for social (gray bars) and isolated (white bars) mice to complete pure tone detection training. Boxes represent upper and lower quartile range, horizontal lines represent median, error bars represent maximum and minimum number of days.

\section{Discrimination of target USVs}

The discrimination performance (percent correct) for all target USVs within a single background USV was compared between mice in the two housing conditions (means are shown in Fig. 6, separately for mice in each housing condition). Both groups were generally good at discriminating between USVs (high percentage of green squares). Percent discrimination varied across combinations from $9.6 \%$ to $100 \%$. The Mann-Whitney $U$ test found no significant differences between socially housed and isolated mice for any USV-USV discrimination combination.

\section{Multidimensional scaling analyses}

Individual matrices were created from each mouse's percent correct discrimination of every USV target from every USV background. These matrices were used to calculate multidimensional maps of USV perception in the two groups of mice (Fig. 7). Due to the asymmetry of the matrices (percent corrects were not equal in corresponding cells above and below the diagonals), the maps were created using a PROXSCAL analysis using the full matrix from each mouse. The two-dimensional maps accounted for $91 \%$ of dispersion in social mice and $90 \%$ of dispersion in isolated mice. In general, for mice in both housing conditions, perceptual maps generally grouped USVs within categories close together, especially the complex and upsweep USVs. There are differences between the two maps, suggesting that overall perception of the USVs differs between the two groups of mice, although that difference cannot be quantified using this technique. Individual weight maps depict the similarity of responses based on mouse identity (Fig. 8). There were no clear separations of mice from the two housing conditions. That is, individual subjects from both groups used similar features of USVs for discrimination.

\section{a Isolated}

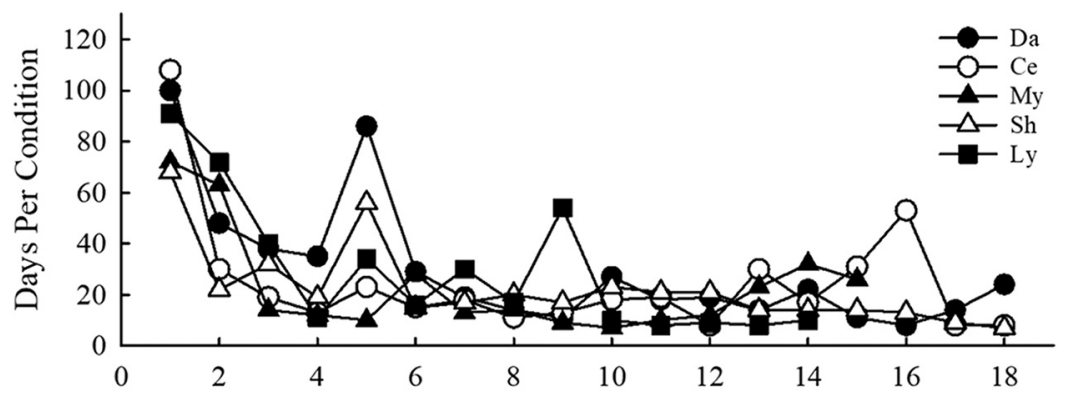

\section{b Social}

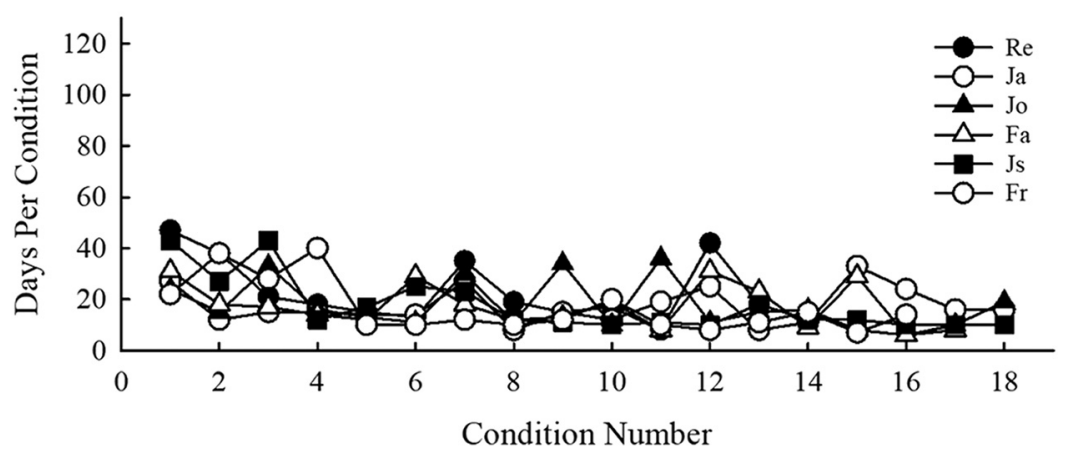

Figure 5. Number of days required for (a) isolated and (b) socially housed mice to complete each of the 18 background USV discrimination conditions. 


\section{a Social}

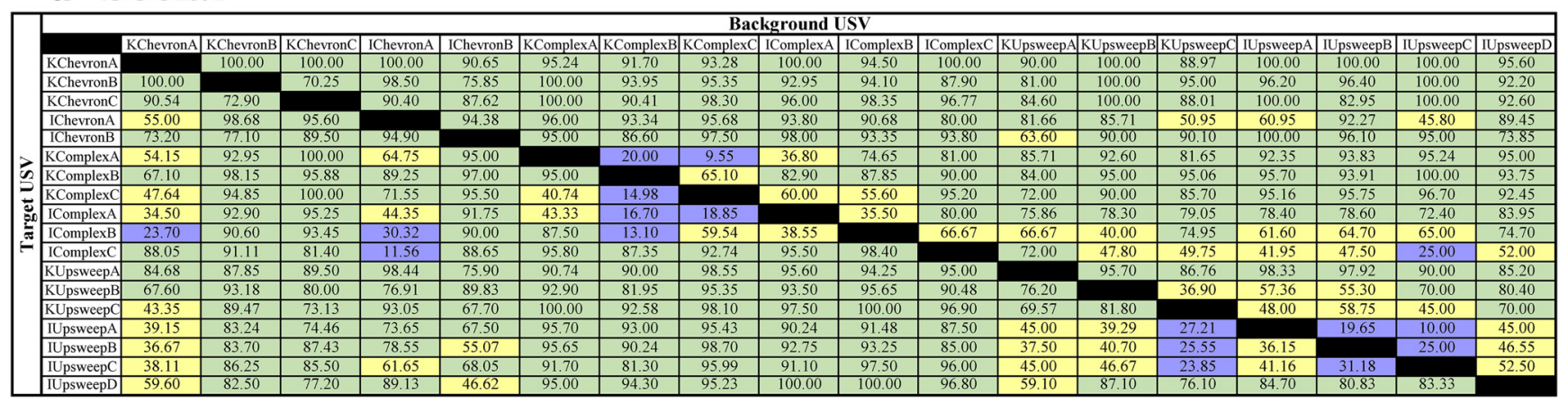

\section{b Isolated}

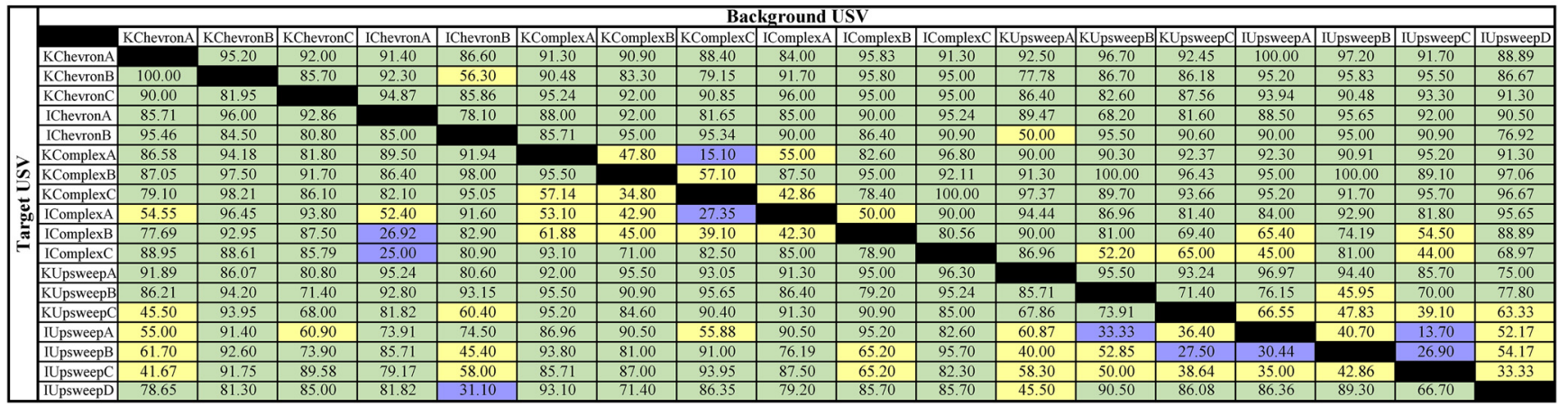

Figure 6. Mean percent correct discrimination values for every background USV against every target USV for social (a) and isolated (b) mice. Percent corrects ranging from $0 \%$ to $33 \%$ are shaded in purple. Percent corrects ranging from $34 \%$ to $66 \%$ are shaded in yellow. Percent corrects ranging from $67 \%$ to $100 \%$ are shaded in green.

\section{Spectrotemporal similarity}

Spectrotemporal similarity was computed using Raven Pro software (v.1.5, Cornell Lab of Ornithology) using the Batch Correlator function, which computed the similarity of the 18 USVs against each other (see Raven Pro 1.4 User's Manual, pp 221-224 for calculation). Similarity ranged on a scale from 0 to 1 , with 0 being not at all similar and 1 being identical. The role of spectrotemporal similarity on discrimination performance was examined using Spearman's rank-order correlation (Fig. 9). There was a significant negative correlation between spectrotemporal similarity and percent discrimination in both so- a Social

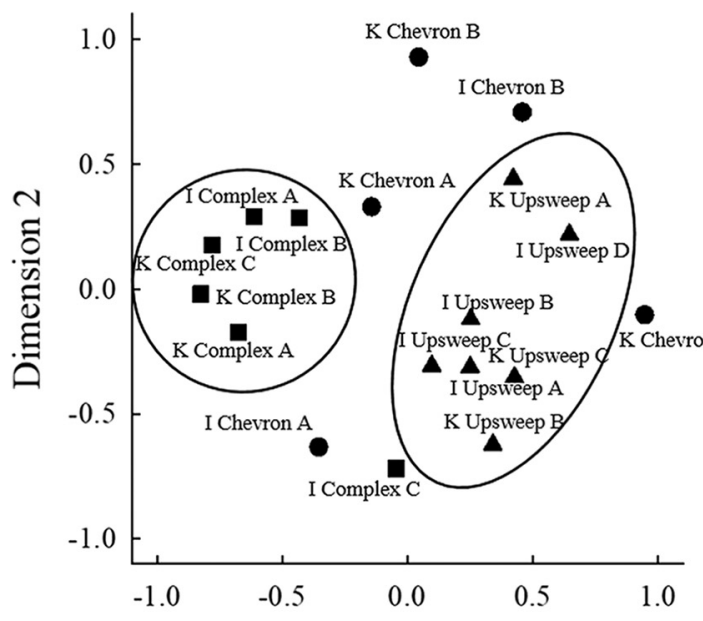

Dimension 1

\section{b Isolated}

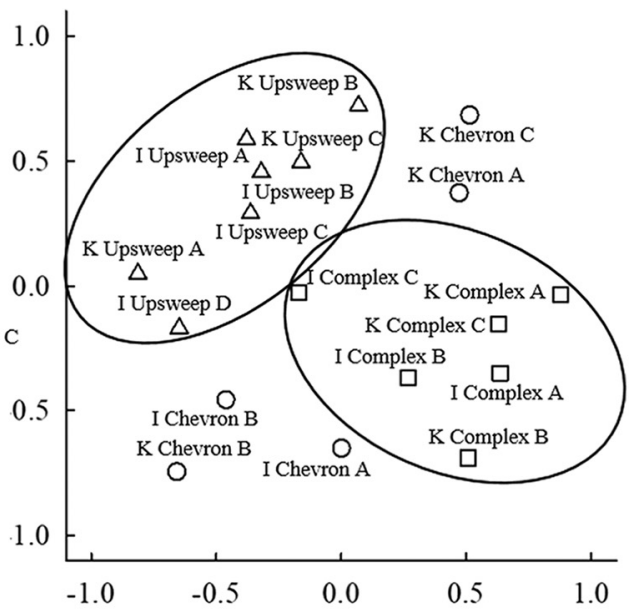

Dimension 1

Figure 7. Multidimensional scaling analysis for (a) social (filled symbols) and (b) isolated mice (open symbols) for chevron (circles), complex (squares), and upsweep (triangles) USVs. Large circles represent researcher-created categories of USVs in similar perceptual spaces. 


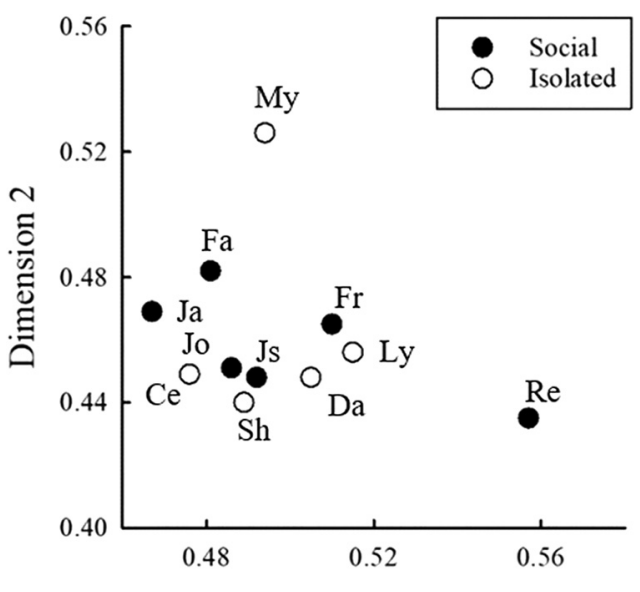

Dimension 1

Figure 8. Map of the individual subject weights of social (filled symbols) and isolated (open symbols) mice that contributed to the multidimensional scaling analysis. Subjects close together used similar attributes when discriminating between USVs.

cially isolated $\left(r_{s}^{2}=-0.320, p<0.001\right)$ and socially experienced mice $\left(r_{\mathrm{s}}^{2}=-0.389, p<0.001\right)$.

\section{Categories of USVs}

For both socially housed and isolated mice, some USVs were difficult to discriminate (e.g., KComplexC vs KComplexA), while other USVs were easy to discriminate (e.g., KChevronA USV targets vs every other USV background). No clear pattern emerged in Figure 6 as to which discriminations were difficult and which were easy, except that discriminations within researcher-defined categories (e.g., complex vs complex) were harder than discriminations across researcher-defined categories (e.g., upsweep vs chevron). When we compared the mean percent discrimination performance for within versus across categories (using the matrices in Fig. 6), this became even more apparent, at least for two of the three categories (complex and upsweep; Fig. 10).

The spectrotemporal correlations in the section above suggest that spectrotemporal similarity plays a role in discriminating between USVs. In Figure 9, the outline color of the symbols represents whether that particular discrimination type was within (purple outlines) or across (green outlines) researcher-defined USV categories. Although discrimination was generally poorer when spectrotemporal similarity was high, Figure 10 shows that this was not the only factor in discriminating between USVs. Most of the lowest percent discrimination values in Figure 9 are outlined in purple, across all spectrotemporal similarities, suggesting categorical perception may also be contributing to performance.

The categorical perception of USVs was further examined using a Mann-Whitney $U$ test for socially housed and isolated mice for each of the three USV categories (Fig. 11). Discrimination of USVs within the same category was significantly worse for socially housed mice for both complex $(U=19,551.500, p<0.001)$ and upsweep $(U=$ $27,654.000, p<0.001$ ) backgrounds. Discrimination was also worse within the same category than between categories in isolated mice for complex $(U=19,749.000, p<$ $0.001)$ and upsweep $(U=37,322.500, p<0.001)$ backgrounds. The pattern of discrimination reversed for chevron backgrounds for both social $(U=32,317.500, p<$ $0.001)$ and isolated mice $(U=17,024.000, p<0.001)$, with mice discriminating targets within the chevron category better than they discriminate chevrons versus USVs from other categories. These results are similar to the multidimensional scaling analyses results, where the mice placed the complex and upsweep USVs into separate perceptual spaces, while the chevrons were located throughout the maps. Thus, it appears that the chevron

\section{a Social}

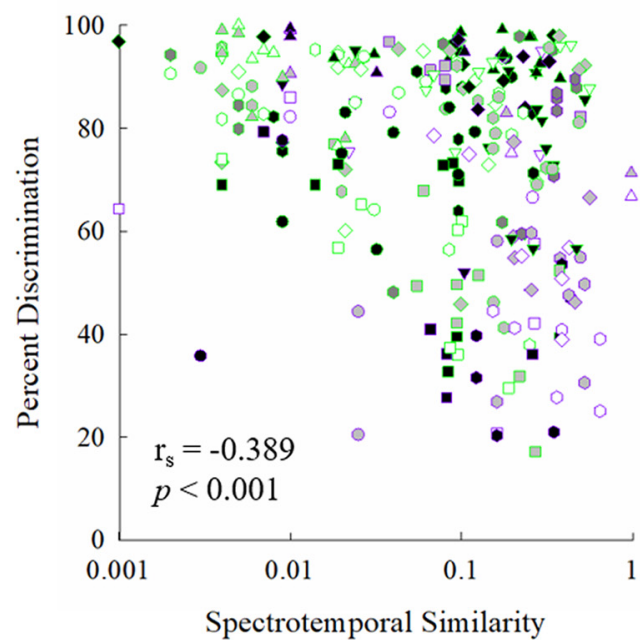

\section{b Isolated}

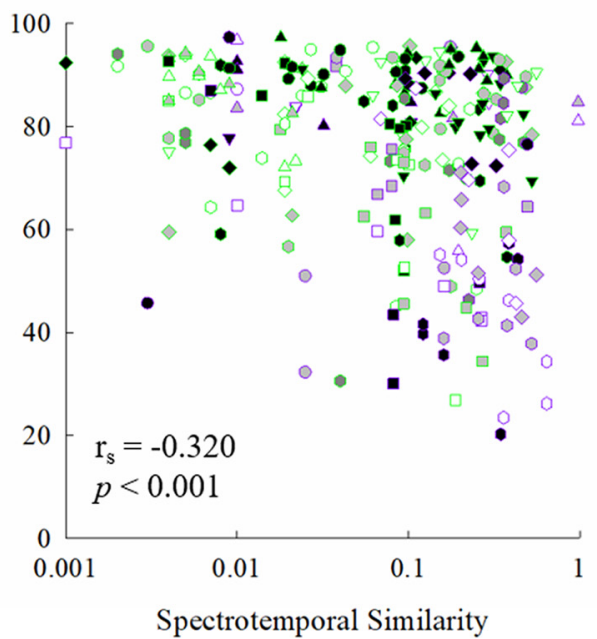

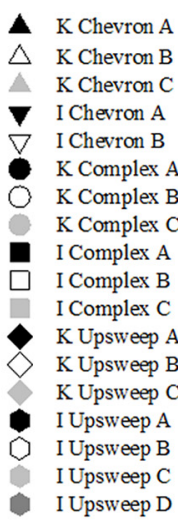

Spectrotemporal Similarity

Figure 9. Percent correct discrimination as a function of spectrotemporal similarity by (a) social and (b) isolated mice. Symbols represent mean discrimination for each target in all 18 conditions. Each symbol represents a different background USV. Symbol edges are purple for within category discriminations and green for across category discriminations. Spearman's rank order correlation coefficients are shown for each group of mice. 


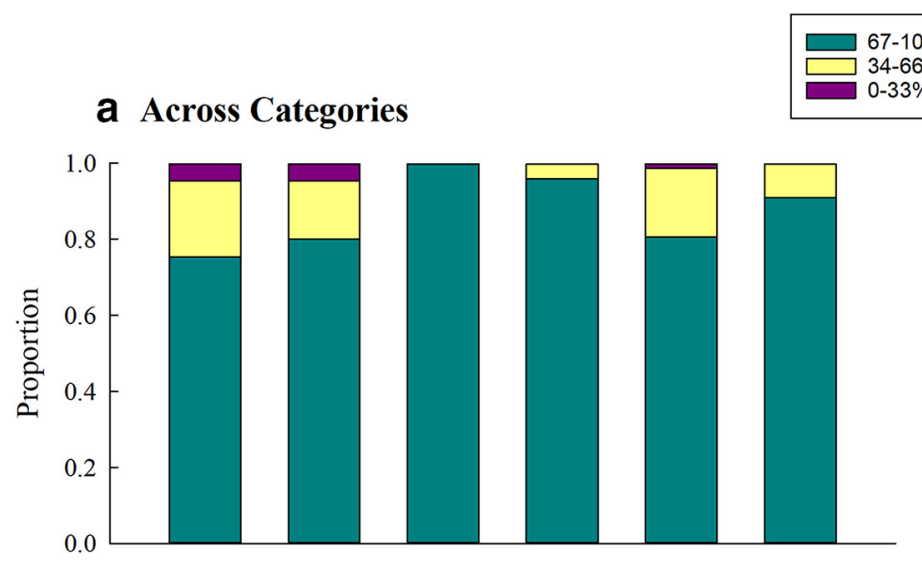

\section{b Within Categories}

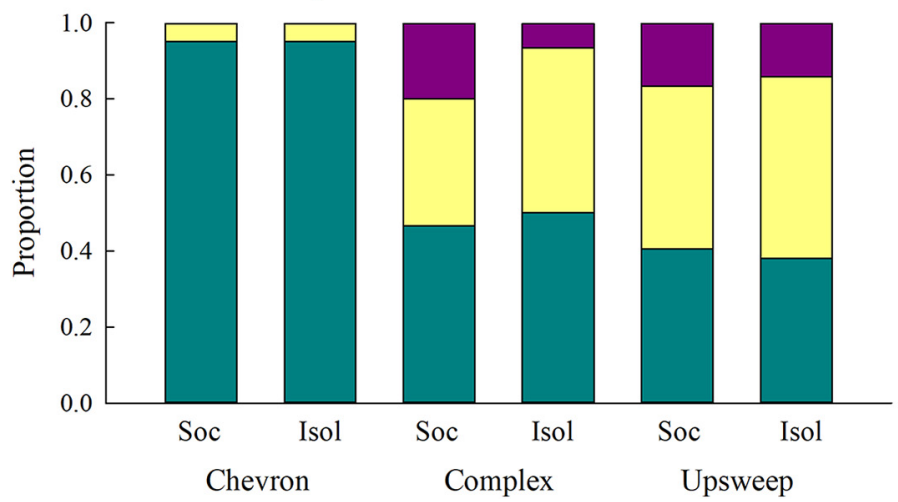

Figure 10. Mean percent discrimination (a) across categories and (b) within categories, separated by USV (chevron, complex, and upsweep) and housing condition (socially housed (Soc) and isolated (Isol)). Green portions of the bars represent the best discrimination abilities (67\% to $100 \%$ ), yellow portions represent medium discrimination abilities (34\% to $66 \%$ ), and pink portions represent poor discrimination abilities (0-33\%).

\section{a Chevron}

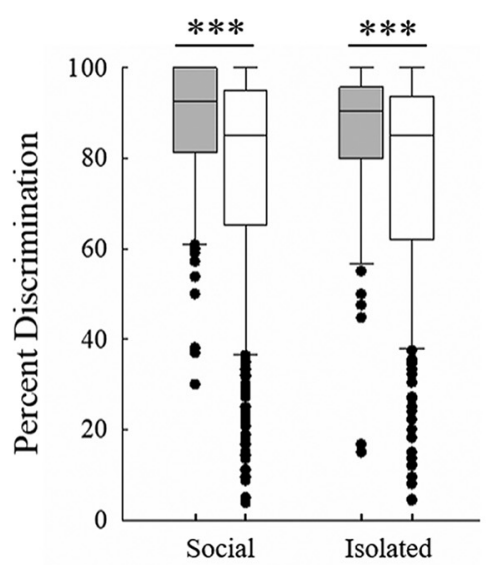

Housing Group b Complex

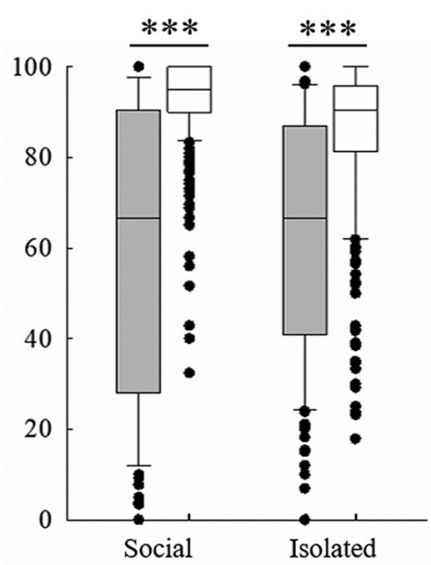

Housing Group c Upsweep

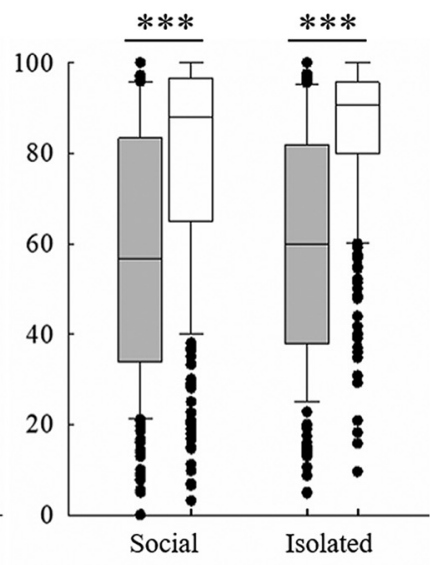

Housing Group

Figure 11. The percentage of correctly discriminated targets from the background USV for within (gray bars) and across (white bars) categories for social and isolated mice tested on (a) chevron, (b) complex, and (c) upsweep USVs. Bars represent upper and lower quartile range of percent discrimination, horizontal lines represent the median, and filled symbols represent data outside the interquartile range; $* * * p<0.001$. 
USVs were not perceived as a single category, while the mice may have perceived the complex and upsweep USVs categorically.

\section{Discussion}

The goal of this experiment was to determine whether the perception of USVs was affected by social isolation in mice. It is unclear whether social isolation caused deficits in learning the operant task using simple auditory stimuli (16-kHz pure tone), but isolated mice required significantly more days to learn the task than socially housed mice. There were early deficits in USV discrimination for mice in both housing groups, which disappeared after a few sessions. This deficit was more pronounced in isolated mice, as they required significantly more days to complete the first USV discrimination condition than socially housed mice. It is worth investigating further whether social isolation alters acoustic processing in general, or whether it could be limited to natural signals, such as USVs. If social isolation produces significant stress or alters motivation levels of the mice, there should be no difference between natural and synthetic signal perception, just differences in task learning. However, the effects of this type of "social buffering" (Sullivan and Perry, 2015) on auditory perception are largely unknown.

The multidimensional scaling analysis (Fig. 7) showed that mice grouped USVs into categories that generally agree with the categories we created for the stimuli in this experiment. It is important to note that the location of each USV relative to all the others is the main point of consideration, and the exact location of the USVs is not relevant for the interpretation of the maps. The greatest differences in the perception of USVs between housing conditions emerged within the chevron category. For both socially housed and isolated mice, chevron USVs were not localized as a single group, but were located between and around the complex and upsweep categories; however, the location of these USVs within the perceptual space was not the same for isolated and socially housed mice. This suggests that isolated and socially housed mice perceived USVs classified as chevrons a priori much differently, despite these USVs having similar spectrotemporal "shapes." In contrast, mice in the two housing conditions showed similar patterns of mapping for the complex and upsweep USVs. The lack of a distinct chevron group in the maps suggests that the criteria used by researchers to define categories are not always accurate; mice may attend to characteristics of the USVs that researchers currently do not take into account when creating categories.

Mice from both housing conditions used similar parameters of USVs to discriminate between stimuli. The dimensions of both perceptual maps were compared to the parameters of the stimuli and general patterns emerged (exceptions to these patterns may exist, and we have no way of knowing from these results whether these are the parameters that the mice are actually using for discrimination). In socially housed mice, dimension 1 of the perceptual map could correspond to the starting frequency of the USVs. The negative side of this dimension corre- sponds to USVs with higher starting frequencies, and the positive end contains USVs with lower starting frequencies. Alternately, dimension 1 could correspond to frequency modulation. The negative side contains "complex" USVs with a larger number of changes in frequency direction within the USV and the positive section contains "simple" USVs with fewer changes in frequency direction across the USV. Dimension 2 may be related to the duration of the stimuli, as USVs with longer durations are generally aligned with the positive portion of this dimension and USVs with shorter durations generally aligned with the negative portion of this dimension. Dimension 1 for isolated mice likely corresponds to the frequency modulation of the USVs. The negative portion of dimension 1 contains simple USVs with fewer changes in frequency direction, whereas the positive portion contains complex USVs with more changes in frequency direction. Dimension 2 in the isolated mice's perceptual map appears to relate to duration, with USVs of shorter durations aligned with the positive portion and longer durations aligned with the negative portion of this dimension. There may be other parameters used by the mice to aid in their discrimination that could align with these dimensions as well.

The results from the two socially housed mice who were new to psychophysical testing (Fr, Js) did not differ from three of the four mice who had previously participated in a USV discrimination task ( $\mathrm{Fa}, \mathrm{Ja}, \mathrm{Jo})$, as shown in the individual weight map (Fig. 8). Additionally, the individual weight map shows that socially housed and isolated mice generally perceived USVs the same way. The majority of mice in both groups were clustered together, providing evidence for similar perceptual patterns for USVs.

This experiment is only the third investigation of USV discrimination using natural stimuli from adult mice and behavioral methods. Using similar methods, Holfoth et al. (2014) found that, in mice, the beginning of the USV was more important for discrimination than the middle or end of the USV, paralleling human word recognition. In the present experiment, the very slight differences in the stimulus presentation rhythm when a background USV was alternated with the target USVs are unlikely to be noticed, as the mice in the Holfoth et al. (2014) experiment showed extremely poor performance ( $\sim 20 \%$ correct) when discriminating a whole USV from the first third of a cropped USV. This was found to be unique to USVs, as discriminating between a USV and a short pure tone was easier (but mice still performed at only 60\% correct). Neilans et al. (2014) found that mice could discriminate between USVs of five categories. The results from Neilans et al. (2014) suggested mice relied on spectrotemporal similarity of USVs to aid in their discrimination. When two USVs were highly spectrotemporally correlated, they were more difficult to discriminate. The current experiment aimed to expand on the results of Neilans et al. (2014) by using multiple USVs per category, as well as to determine whether social experience changes how mice rely on spectrotemporal similarity to discriminate between USVs. Mice in both housing conditions showed a negative correlation between spectrotemporal similarity and USV discrimination, agreeing with the results of Neilans et al. 
(2014). In socially isolated mice, spectrotemporal similarity accounts for $32.0 \%$ of the variance observed in discrimination performance. Similarly, spectrotemporal similarity accounts for $38.9 \%$ of the variance in socially housed mice's discrimination ability, slightly more than in isolated mice. This suggests that mice rely on spectrotemporal similarity, at least in part, to help discriminate between USVs.

Finally, this experiment was the first to test whether mice perceive their USVs in accordance with the researcher-defined categories that are found throughout the literature. Categorical perception is characterized by poor discrimination of within-category stimuli and better discrimination of across-category stimuli. We investigated whether mice showed this pattern for discrimination of researcher-defined USV categories. Mice in both housing conditions showed increased discrimination performance when the target and background USVs were in different categories compared to when both target and background USVs were within the same category (Figs. 9-11). Although it has previously been demonstrated that maternal female mice show categorical perception of pup calls (Ehret and Haack, 1981; Ehret, 1992), this is the first step in demonstrating that mice may show categorical perception of adult USVs, providing further evidence that mice are able to use their USVs to communicate important information. Although much more investigation is required to determine how USVs are used in adult communication, it is clear that mice are able to discriminate between USVs, even those within the same researcher-defined categories (e.g., complex vs complex). The results of this experiment provide evidence that mice could be using USVs for communication, since the first criterion is discriminating between different USVs. Further, both the categorical perception analyses and the multidimensional scaling analyses suggest that some researcher-defined categories of USVs are better than others. Discriminating among chevron USVs did not follow the same trend as discrimination of the other two categories.

The findings of the present experiment demonstrate how perception of communication signals is and is not affected by social experience. Socially housed and isolated mice relied, at least in part, on different parameters of USVs to aid in discrimination. This difference is illustrated by dissimilarities between the perceptual maps of mice from the two housing conditions. The results suggest that socially housed mice are probably more attentive to specific features of USVs (e.g., start frequency) compared to isolated mice, who attended to more global features of the targets (e.g., duration, overall frequency modulation). The categorical perception analyses suggest that any differences in the perception of USVs between housing groups are minor, and do not affect the general classification of various acoustic signals. The differences in results between the complex and upsweep USVs (seemingly accurately defined mouse perceptual categories) and the chevron USVs (seemingly inaccurately defined mouse perceptual categories) suggest that mice may be attending to characteristics present in USVs that humans are unable to recognize.

\section{References}

Chabout J, Sarkar A, Dunson DB, Jarvis ED (2015) Male mice song syntax depends on social contexts and influences female preferences. Front Behav Neurosci 9:76.

Chabout J, Sarkar A, Patel SR, Radden T, Dunson DB, Fisher SE, Jarvis ED (2016) A Foxp2 mutation implicated in human speech deficits alters sequencing of ultrasonic vocalizations in adult male mice. Front Behav Neurosci 10:197.

Cohen L, Rothschild G, Mizrahi A (2011) Multisensory integration of natural odors and sounds in the auditory cortex. Neuron 72:357369.

Dent ML, Screven LA, Kobrina A (2018) Hearing in rodents. In: Rodent bioacoustics (Dent ML, Fay RR, Popper AN, eds). Cham: Springer Nature Switzerland.

Ehret G (1992) Categorical perception of mouse-pup ultrasounds in the temporal domain. Anim Behav 43:409-416.

Ehret G, Haack B (1981) Categorical perception of mouse pup ultrasound by lactating females. Naturwissenschaften 68:208209.

Hammerschmidt K, Radyushkin K, Ehrenreich H, Fischer J (2012) The structure and usage of female and male mouse ultrasonic vocalizations reveal only minor differences. PLoS One 7:e41133.

Heffner HE, Heffner RS (2001) Auditory communication among adults. In: Handbook of mouse auditory research (Willott JF, ed), p 19. Boca Raton: CRC Press.

Heffner HE, Koay G, Heffner RS (2008) Comparison of behavioral and auditory brainstem response measures shift in rats exposed to loud sound. J Acoust Soc Am 124:1093-1104.

Holfoth DP, Neilans EG, Dent ML (2014) Discrimination of partial from whole ultrasonic vocalizations using go/no-go task in mice. J Acoust Soc Am 136:3401-3409.

Klink KB, Bendig G, Klump GM (2006) Operant methods for mouse psychoacoustics. Behav Res Methods 38:1-7.

Liu RC, Schreiner CE (2007) Auditory cortical detection and discrimination correlates with communicative significance. PLoS Biol 5:e173.

Neilans EG, Holfoth DP, Radziwon KE, Dent ML (2014) Discrimination of ultrasonic vocalizations by CBA/CaJ mice (Mus musculus) is related to spectrotemporal dissimilarity of vocalizations. PLoS One 9:e85405.

Neunuebel JP, Taylor AL, Arthur BJ, Egnor SER (2015) Female mice ultrasonically interact with males during courtship displays. Elife 4:e06203.

Okanoya K, Screven LA (2018) Rodent vocalizations: adaptations to physical, social, and sexual factors. In: Rodent bioacoustics (Dent ML, Fay RR, Popper AN, eds). Cham: Springer Nature Switzerland.

Portfors CV (2007) Types and functions of ultrasonic vocalizations in laboratory rats and mice. J Am Assoc Lab Anim Sci 46:28-34.

Portfors CV, Roberts PD, Jonson K (2009) Over-representation of species-specific vocalizations in the awake mouse inferior colliculus. Neuroscience 162:486-500.

Scattoni ML, Ricceri L, Crawley JN (2011) Unusual repertoire of vocalizations in adult BTBR $\mathrm{T}+\mathrm{tf} / \mathrm{J}$ mice during three types of social encounters. Genes Brain Behav 10:44-56.

Screven LA, Dent ML (2018) Preference in female laboratory mice is influenced by social experience. Behav Processes 157:171-179.

Screven LA, Dent ML (2019) Social isolation produces no effect on ultrasonic vocalization production in adult CBA/CaJ mice. PLoS One 14:e0213068.

Sewell GDS (1972) Ultrasound and mating behavior in rodents with some observations on other behavioural situations. Animal Behav 28:149-164.

Sullivan RM, Perry RE (2015) Mechanisms and functional implications of social buffering in infants: lessons from animal models. Soc Neurosci 10:500-511. 